\title{
Deformation Velocity Survey in Mortar and Cement Paste Specimens Subjected to External Aggressive Attacks
}

\author{
Cristina Tedeschi and Elsa Garavaglia \\ Department of Civil and Environmental Engineering, Politecnico di Milano, Milan, Italy. \\ cristina.tedeschi@polimi.it, elsa.garavaglia@polimi.it
}

\begin{abstract}
The durability of concrete depends not only on the composition of the material and its resulting porosity, but also on the environmental conditions. Contact between concrete or cement mortars with water and soils containing sulphates, of a natural, biological origin or deriving from pollution, can activate chemical reactions in the cement paste inducing an expansion and degradation of the concrete. To evaluate the behaviour of the cementitious material subjected to various external aggressive attacks, prismatic samples of cement paste and mortar samples produced with the same binder (Portland cement) were immersed in demineralised water and in various solutions containing $5 \%$ and $10 \%$ sodium sulphates. Their expansion was monitored over time by a direct length measurement. This paper shows the results achieved in a rather long laboratory investigation and a first hypothesis has been formulated on the possible expansion rate in the first 56 test days and in the following 850 days.
\end{abstract}

Keywords: Cement Mortar, Aggressive Environment, Sodium Sulphate Attacks, Experimental Tests, Deformation Velocity.

\section{Introduction}

The durability of a concrete structure represents the ability to withstand aggressive environmental stresses, therefore it depends not only on the composition of the material and the resulting porosity, but also on environmental conditions. The different causes of damage to concrete, can be divided into different types depending on whether the aggressive action involves one or more components of the conglomerate (cement paste, aggregate or metallic reinforcement).

In particular, when a concrete structure comes into contact with waters or soils rich in sulphates, a series of complex chemical reactions can occur the sulphates which propagate inside the material and aluminates of cement paste, leading to the formation of leading to the formation of gypsum, secondary ettringite, and in some cases, thaumasite with the consequent expansion and formation of cracks and expulsion of parts of concrete. These phenomena are collected in the general term of the external sulphate attack.

Various researches have been carried out on this subject, many of which were dedicated to the description of sulphate attack from an experimental point of view. There are numerous experimental campaigns carried out on cement paste, mortar and concrete samples, however the results depend strongly on the test conditions, the cement used and the aggregates and their particle size distribution.

On this topic there are researches in the literature that for the most part focus on the evaluation of durability and the evolution of damage (Anzani et al., 2018, Garavaglia et al., 2018, Garavaglia et al., 2017, Garavaglia et al., 2016, Anzani et al, 2009, Garavaglia et al., 2008, Tedeschi and Garavaglia, 2019, Cefis et al. 2015, Tedeschi et al. 2017). 
In this paper, the results of an ongoing experimental campaign are reported, carried out on prismatic samples of cement paste and mortar. An objective of this experimental program is also to highlight the role of the aggregate on the degradation phenomenon, for this reason samples of cement mortar were prepared with two different types of aggregate.

To evaluate the behaviour of the cement material subjected to various external aggressive attacks, laboratory tests were carried out during which cement paste samples (P-CEM), mortar samples with normalised sand (M-nor) and samples of mortar with aggregate (M-agg), were subjected to immersion cycles, thus simulating three different aggressive attacks: from demineralised water, from sodium sulphate at $5 \%$ salt concentration and from sodium sulphate at $10 \%$ concentration. The expansion was monitored over time by direct length measurements.

The tests were performed in accordance with the UNI EN 12617-4: 2003, but they lasted longer in time in order to verify any changes in behaviour over time.

The results obtained in the 889 days are shown as the variational behaviour of the deformation (shrinkage/expansion) recorded, but also in terms of deformation velocity, variable at each instant of the test process and function of the deformation value recorded.

There are not many approaches to this topic in the literature. The topic investigated is the transmission of waves in the material (Tseng et al., 1999, Boyd and Ferraro, 2005), not the deformation velocity of materials subject to degradation. A similar approach is present in Zhanga et al., 2012, which stimulates the authors to continue on this path.

Future research developments will be oriented towards probabilistic modelling of the detected behaviours.

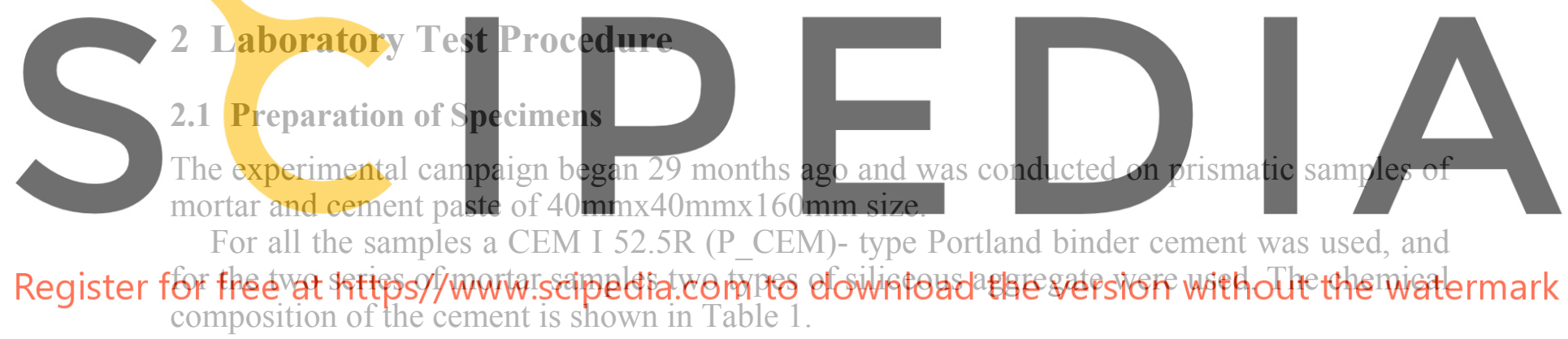

Table 1. Chemical composition of cement.

\begin{tabular}{cccc}
\hline Cement & C3A & C4AF & SO3 \\
\hline CEM I 52.5R & $4.22 \%$ & $5.16 \%$ & $3.61 \%$ \\
\hline
\end{tabular}

The named samples (M_agg), were prepared with a siliceous aggregate for concrete, but used by eliminating the aggregates greater than $2 \mathrm{~mm}$ by sieving, while the named prisms (M_nor), were prepared with standardised sand used for the certification of mortars (EN 1015-1).

To evaluate the behaviour of the cementitious material subjected to different external aggressive attacks, three prisms for each type of mixture were prepared, and laboratory tests were performed during which the prismatic samples were immersed respectively in deionized water and in a solution of 5 and $10 \%$ sodium sulphate. Once hardened, the samples were subjected to measurement to evaluate their variations in length, so as to be able to study their overall expansion, but above all to evaluate the trend and the degradation mechanisms over time. 


\subsection{Test Procedure}

The determination of the expansion was determined according to the standard EN 12617-4: 2003, using an uncontrasted linear measurement method after the immersion in water of prismatic of $40 \mathrm{mmx} 40 \mathrm{~mm} \times 160 \mathrm{~mm}$ size specimens, from $24 \mathrm{~h}$ after the casting.

The samples were prepared/made in a climatic chamber at temperature $\mathrm{T}=20^{\circ} \mathrm{C}$ and relative humidity $\mathrm{RH}=65 \%$ and subsequently placed in a climatic chamber at $\mathrm{T}=20^{\circ} \mathrm{C}$ and relative humidity $\mathrm{RH}=90 \%$. After 24 hours from the preparation, the specimens were removed from the moulds and completely immersed in pure water (double-distilled) and in a solution with a concentration of $5 \% \mathrm{Na}_{2} \mathrm{SO}_{4}$ and $10 \%$ sodium sulphate.

The prisms were periodically extracted from the solutions, in order to be able to measure the variations in weight and length. Before the initial measurement, the adhesion of the measuring pins was verified. Table 2 indicates the codes of the samples and their corresponding test conditions. In Figure 1, Samples during the measurement phase; b) Samples during the last curing period.

Table 2. Name of the individual specimens and seasoning conditions.

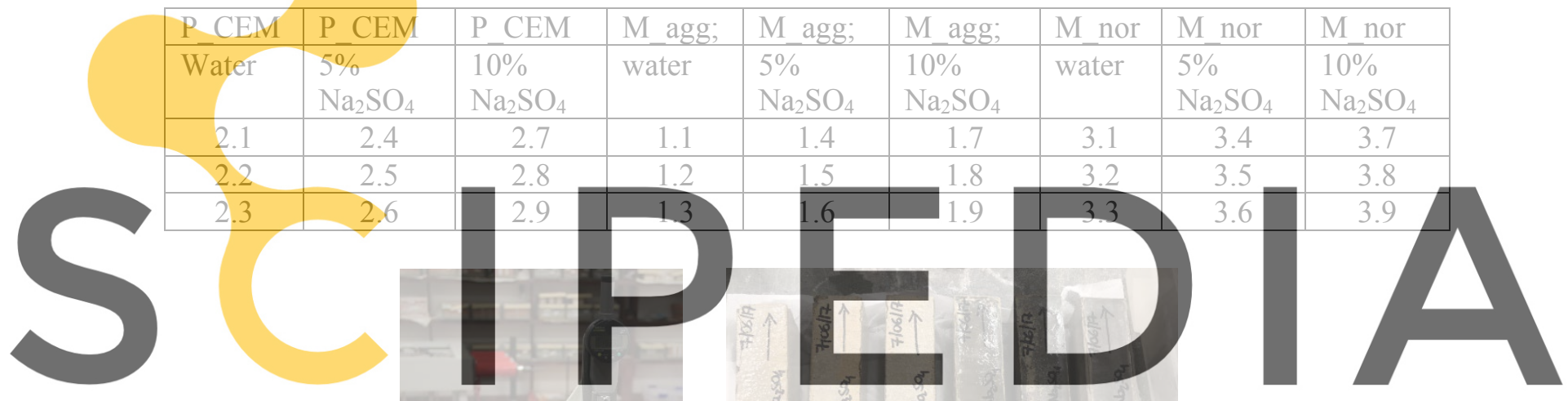

Register for free at https//www.scipedia.com to download the version without the watermark

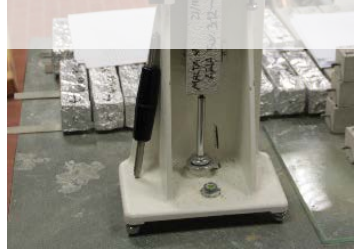

a)

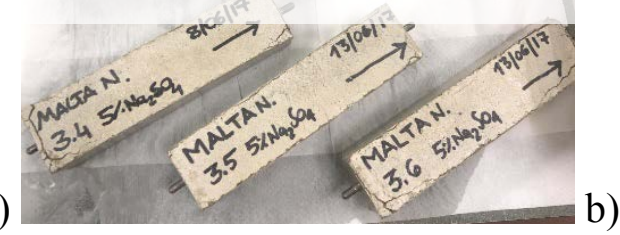

Figure 1. a) Samples during the measurement phase; b) Samples during the last curing period.

\section{Deformation Survey}

\subsection{Deformation Measurement}

In compliance with the standards required in UNI EN 12617-4:2003 the variation in length was calculated in relation to $L_{0}$ length detected after 24 hours of immersion of the specimens in water:

$$
\Delta L_{i}=\left(L_{i}-L_{0}\right)
$$


where: $\Delta L_{i}$ is the variation of the length at time $t_{i} ; L_{i}$ is the length of the specimen at time $t_{i}$; $L_{0}$ is the initial length of the specimen after 4 hours of immersion.

The deformation $\varepsilon_{i}$ at each instant of measurement, $t_{i}$, is thus expressed by the formula:

$$
\varepsilon_{i}=\frac{\Delta L_{i}}{L_{g}}
$$

where $\Delta L_{i}$ is the variation of length expressed by (1) and $L_{g}$ is the verified length of the specimen before immersion (in the case studied $160 \mathrm{~mm}$ ).

\subsection{Observations on the Results Obtained}

Figures 2 and 3 show the trend of the average deformations recorded for the tested specimens. In these figures, the deformation values are compared in the three aggressive situations imposed on the test: immersion in water, and immersion in a saline solution with a 5\% and $10 \%$ concentration, for each type of mixture.

The deformation recorded in the first 56 days of testing shows an initial shrinkage (indicated with a negative numbering in figure 2) more evident in P-CEM specimens.

The continuation of the phenomenon seems to be more rapid for the P-CEM and slower and almost stationary in the M-agg and M-nor. In P-CEM the salt concentration of 5\% seems to trigger a process of deformation greater than the processes caused by demineralised water and by the salt concentration of $10 \%$. This is probably due to the porosity of the material. (Fig. 2 a),
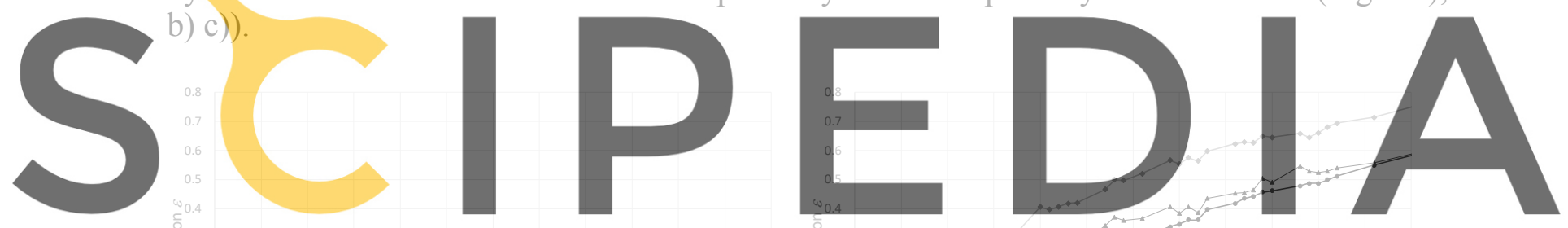

Register för free at https//www.scipedia.com to download the version without the watermark

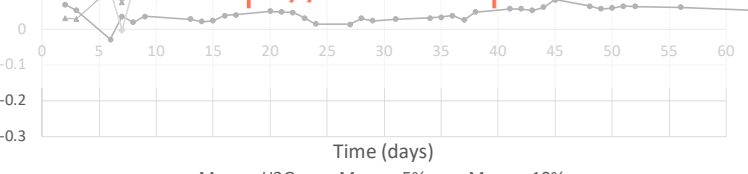

b)

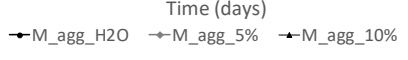

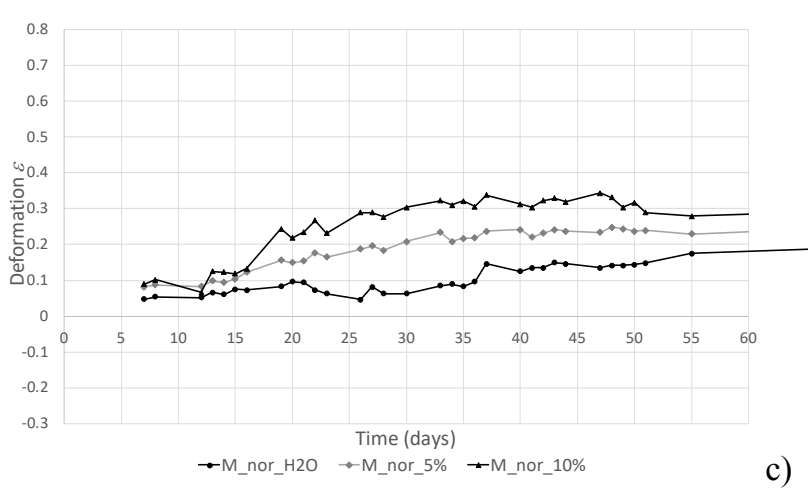

Figure 2. Comparison of the variation of deformation in the first 56 days of testing, referring to the three aggressive agents tested and the mixtures: a) M_agg, b) P_CEM and c) M_nor. 
Figure 3 shows the same comparisons but for the entire testing period ( 889 days).

In this case it is interesting to note that after an initial rather rapid shrinkage of the P-CEM specimens, it stabilises and becomes constant for the entire testing period and for all three aggressive agents. It is not excluded that the continuation of the same test does not lead to different results that are for now unexpected.

Whereas for the M-agg and M-nor mixtures and for the salt concentrations at 5\% and $10 \%$ after the $500^{\text {th }}$ test day there is an increase in the deformation, perhaps as a consequence of initial cracking of the material for specimens immersed in water, instead, there is an almost constant behavior.

This result indicates that the formation of salt crystals inside of less consolidated pastes does not cause serious problems because they fill the pores and interstices without causing serious pressure on the surrounding material. Over time crystals deposit and during the subsequent immersions they do not have the time to melt, therefore the new attack increases the salt volume and the pressure on the material thus producing micro-fractures which accentuate the deformation, furthermore these results show how the addition of aggregate to the cement paste mixture changes the deformation behaviour of the material.
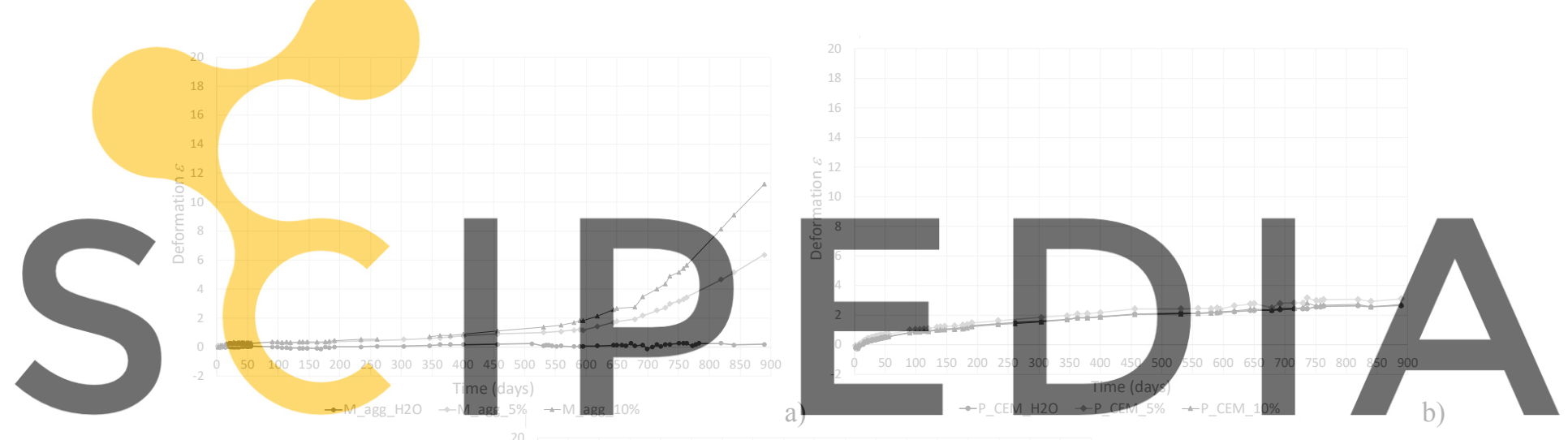

Register for free at https//www.scipedia.com to download the version without the watermark

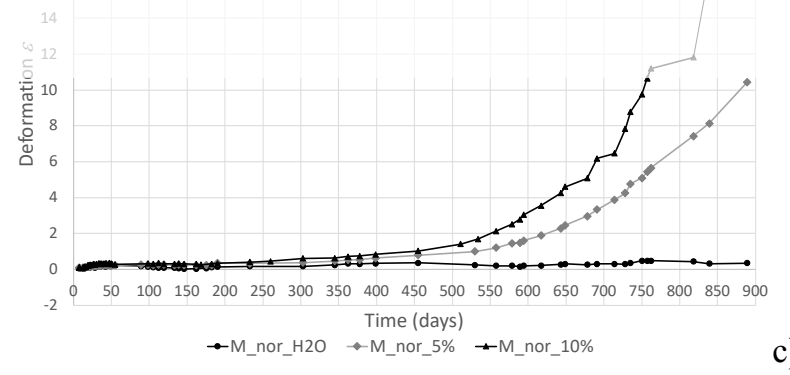

Figure 3. Comparison of the variation of deformation in the 889 testing days, referred to the three aggressive agents tested and the mixtures: a) M_ag: b) P_CEM and c) M_nor.

\subsection{Deformation Velocity Survey}

From the test procedure adopted and from the results obtained from the quantification of the deformation induced by sulphates attack dissolved in different concentration, the survey and the calculation of velocity were carried out and with which the deformation phenomenon occurred. 
The quantification of velocity $\mathbf{v}_{\mathbf{i}}$, relative to each testing cycle, follows the classical definition as reported below:

$$
\mathbf{v}_{i}=\frac{\varepsilon_{i}}{\left(t_{i}-t_{(i-1)}\right)}
$$

where: $\varepsilon_{i}$ is the deformation defined in (2); $t_{i}$ is the instant of testing (measured in days) and $t_{i-1}$ is the previous instant.

Equation 3 allows to evaluate the variation of deformation velocity instant by instant and to formulate hypotheses on the future of the investigated phenomenon.

\subsection{Observation of the Results Obtained.}

The calculation of the variations in velocity was performed for all three mixtures investigated in the test (M_agg; P_CEM and M_nor), both for samples immersed in demineralised water (H2O) and for those with a salt concentration of $5 \%$ and $10 \%$. The results obtained show interesting aspects that characterise the evolution of the deformation phenomenon.

At first the evolution of the phenomenon was considered in the first 56 days of tests. Figure 4 shows the comparison between the behaviour of the samples at the three different stresses for each type of mixture.

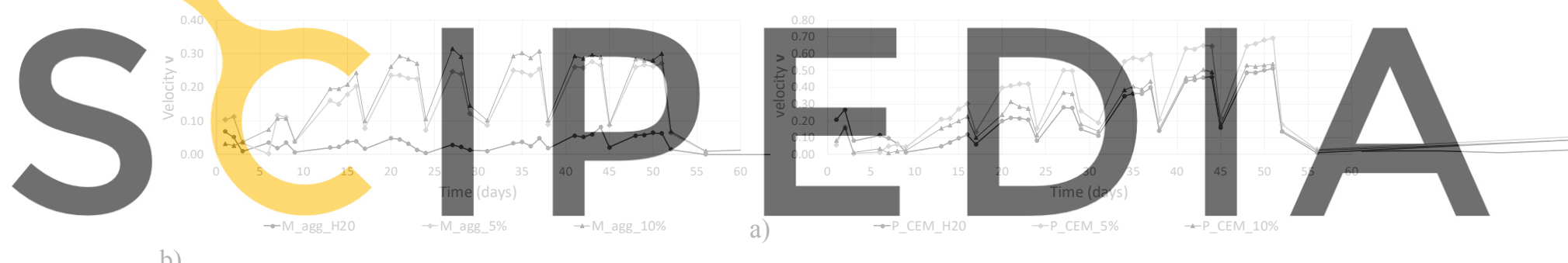

Register for free at https//www.scipedia.com to download the version without the watermark

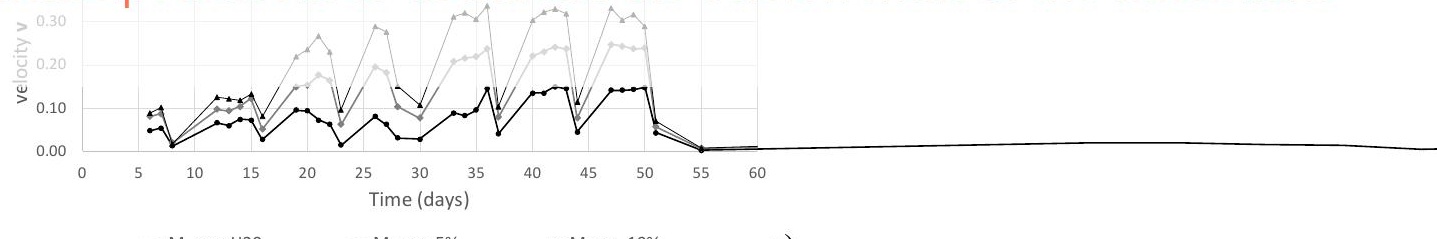

$\rightarrow$ M_nor_H2O

$\rightarrow-M \_n o r \_5 \%$

$\rightarrow-M$ nor_ $10 \%$

c)

Figure 4. Deformation velocity comparison vs. time for the first 56 days of testing referring to the three aggressive agents tested and for the mixtures: a) M_agg, b) P_CEM and c) M_nor.

Figure 4 clearly shows that for all the mixtures there is a cyclic repetition of velocity increase followed by a stasis phase and subsequently by a velocity decrease phase. This trend seems to be associated with the deformation phases (Figure 2 and Figure 3): when the almost stationary deformation phase ends there is a slowing down of the process which then resumes decisively and with an increase in velocity in the next phase. This behaviour is more regular and marked for the P-CEM mixture.

Figure 5 shows, instead, the trend of the deformation velocity, $\mathbf{v}$, for longer test periods. 
Here there is a material compromise after 500 days. Test after test, for all the mixtures there is an increase in velocity, a more evident behaviour for the mixtures subjected to saline aggression.

These velocity increases are followed by decreases and subsequent increments. These variations appear to continue to occur every 30 to 70 days. This cyclicality occurs already after the $100^{\text {th }}$ day of the testing.

From the comparison between Figure 4 and Figure 5, the relationship between the increase in deformation velocity and important changes in the deformation phase is even more evident.
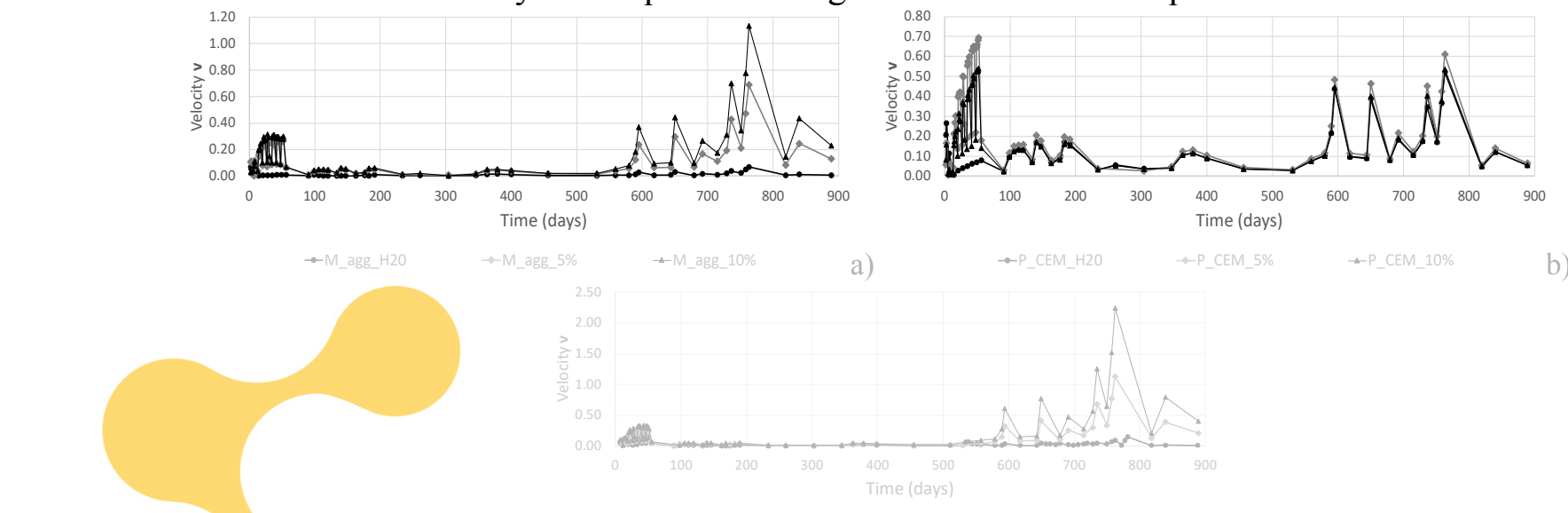

a)

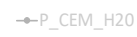

b)
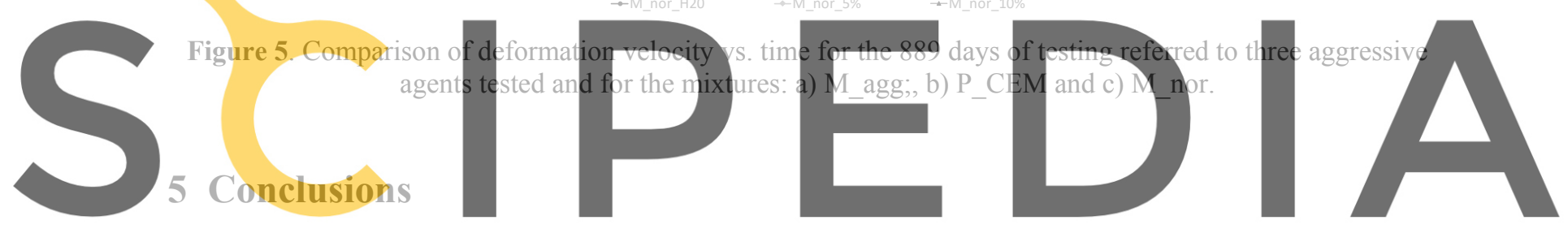

The laboratory tests applied to specimens of different mixtures and subjected to simulations of

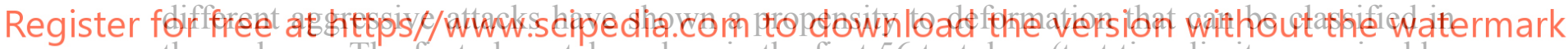

three phases. The first phase takes place in the first 56 test days (test time limit recognised by

the legislation). During this phase the velocity shows a periodicity of behaviour that seems to

be correlated to the occurrence of a greater deformation after a phase of relative stability; this phase is weekly. The next phase shows a constant deformation behaviour. However, in this phase the velocity shows a certain periodicity and the frequency of the periodicity is in this case variable between 30 and 70 days.

The third phase takes place after the 500-day testing and is typical of the behaviour of saline attacks for M-agg and M-nor. It shows a rather variable deformation velocity (Figure 5a and c), but always with a certain cyclical behaviour, which is matched by a decisive increase in deformation behaviour (Figure $3 \mathrm{a}$ and $\mathrm{c}$ ). Moreover, Figure 3 highlights the different deformation behaviour of P-CEM cement paste compared to M-agg and M-nor mixtures. It is possible that this behaviour is associated with the occurrence of a start of detachment of the material now stressed by the presence of salt crystals inside it. This aspect will be investigated. It is the intention of the authors to proceed with the development of a mechanical test that may monitor this phenomenon.

As for the P-CEM mixture the deformation velocity (Figure $5 b$ ) shows the same behaviour recorded for the other mixtures, but with lower values. This is certainly due to a more regular deformation behaviour (Figure 3b). 


\section{ORCID}

Cristina Tedeschi: http://orcid.org/0000-0002-1718-1632

Elsa Garavaglia: http://orcid.org/0000-0002-7448-8374

\section{References}

Anzani A., Cardani G., Condoleo P., Garavaglia E., Saisi A., Tedeschi C., Tiraboschi C. and Valluzzi M.R. (2018). Understanding of historical masonry for conservation approaches: the contribution of Prof. Luigia Binda to research advancement, in Topical Collection 50 years of Materials and Structures, Mater Struct. (2018) 51:140, 1-27.

Anzani A., Garavaglia E. and Binda L. (2009). Long-term damage of historic masonry: a probabilistic model, Construction and Building Materials, Elsevier, 23(2), 713-724 ISSN 0950-0618 (Print).

Boyd A. J. and Ferraro C. C. (2005). Effect of Curing and Deterioration on Stress Wave Velocities in Concrete, Journal of Materials in Civil Engineering, 17(2), 153-158, ASCE, ISSN 0899-1561/2005/2.

Cefis N., Comi C. and Tedeschi C. (2015). Modellazione del degrado nel calcestruzzo dovuto a formazione di ettringite secondaria. In: Memorie estese XII Congresso dell'Associazione Italiana di Meccanica Teorica e Applicata. p. 488-497, ISBN: 978-88-97752-55-4, Genova, Italy, 14/9/2015-17/9/2015.

EN 1015-1:(2006). Methods of test for mortar for masonry - Part 1: Determination of particle size distribution (by sieve analysis).

EN 12617-4:(2003). Product and Systems for the protection and repair of concrete structures. Test Methods Determination of shrinkage and expansion.

Garavaglia E., Anzani A., Binda L. and Cardani G. (2008). Fragility curve probabilistic model applied to durability and long term mechanical damages of masonry. Materials and Structures/Matériaux et Constructions, RILEM Pub. s.a.r.1., Springer Ed., 41(4), 733-749. ISSN 1359-5997 (Print).

Garavaglia E., Basso N. and Sgambi L. (2018). Probabilistic life-cycle assessment and rehabilitation strategies for deteriorating structures: a case study. International Journal of Architecturat Heritage, 12(6), 2018,981-996.

Garavaglia E., Tedeschi accelerated decay by salt Building Materials and $c$ Publication S.A.R.L. Garavaglia E., Tedeschi $\mathrm{C}$., Perego $\$$. and Valluzzi $\mathrm{M}$.
by salt crystallization in fiber reinforced clay brick
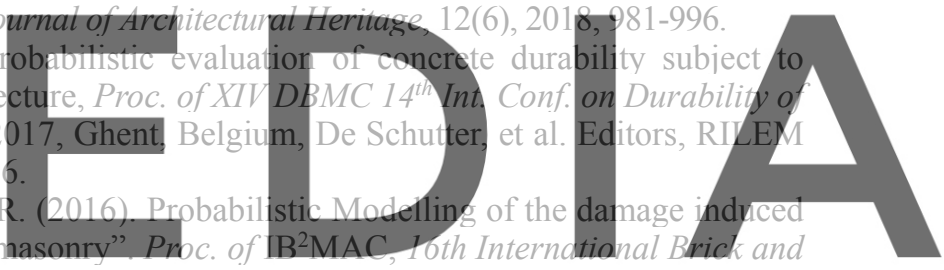
Block Masonry Conference, Trends, Innovations and Challenges, Padova, Italy, June, 26-30, 2016, C. Modena,

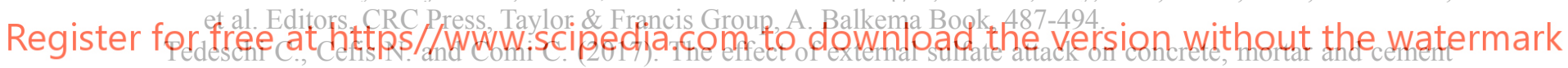
paste, Proc. of XIV DBMC 14th Int. Conf. on Durability of Building Materials and Components. May, 29-31, 2017, Ghent, Belgium, De Schutter, et al. Editors, RILEM Publication S.A.R.L., Paris, PRO 107, USB, $225-236$.

Tedeschi C. and Garavaglia E. (2019). A probabilistic approach to investigate the physical compatibility between bedding and re-pointing mortars, Pro. of SMSS 2019 - RILEM International Conference Sustainable Materials, Systems and Structures, 18-22 March, 2019, Rovinj, Croatia, Bričević et al. Editors, RILEM Publication S.A.R.L., PRO 128, Vol.3, CD-ROM, 700-707.

Tseng W.-T., Chin J.-H. and Kang L.-C. (1999). A Comparative Study on the Roles of Velocity in the Material Removal Rate during Chemical Mechanical Polishing, Journal of The Electrochemical Society, 146 (5), $1952-$ 1959, S0013-4651(97)11-028-X CCC.

Zhanga Y., Abrahama O., Grondinb F., Loukilib A., Tournatc V., Le Duffd A., Lascoupe B. and Duranda O. (2012). Study of stress-induced velocity variation in concrete under direct tensile force and monitoring of the damage level by using thermally-compensated Coda Wave Interferometry, Ultrasonics 52(8), 1038-1045. 